\title{
Tangence
}

\section{Romans pour l'adolescence et intertextualité : les figures de l'écrit comme procédé de représentation du sujet féminin}

\section{Lucie Guillemette}

Numéro 67, automne 2001

L'écriture pour la jeunesse : de la production à la réception

URI : https://id.erudit.org/iderudit/009618ar

DOI : https://doi.org/10.7202/009618ar

Aller au sommaire du numéro

\section{Éditeur(s)}

Presses de l'Université du Québec

ISSN

0226-9554 (imprimé)

1710-0305 (numérique)

Découvrir la revue

Citer cet article

Guillemette, L. (2001). Romans pour l'adolescence et intertextualité : les figures de l'écrit comme procédé de représentation du sujet féminin. Tangence, (67),

96-111. https://doi.org/10.7202/009618ar
Résumé de l'article

Résumé

Cette étude examine des romans pour la jeunesse de Paule Daveluy (1962-1964), de Michelle Marineau (1988-1989) et d'Anique Poitras (1993-1998),

dont les jeunes héroïnes sont enga- gées dans un processus d'écriture. L'utilisation que font ces écrivaines du journal fictif comme procédé de représentation des protagonistes féminines demeure révélatrice d'un processus d'individuation généré par les figures de l'écrit associées ellesmêmes à des lieux du savoir. Ces romans destinés à un public adolescent préfigurent et actualisent tout à la fois les croisements littéraires du postmodemisme et du féminisme à travers les for- mes discursives de l'intertextualité liées à la pratique autorepré- sentative d'un sujet féminin, laquelle suscite un dialogue fécond entre des textes de provenance générique diverse afin de déve- lopper ses propres systèmes de références. 


\title{
Romans pour l'adolescence et intertextualité : les figures de l'écrit comme procédé de représentation du sujet féminin
}

\author{
Lucie Guillemette, \\ Université du Québec à Trois-Rivières
}

Le roman destiné aux adolescents et aux adolescentes invite à une pluralité de lectures, dans la mesure où les problématiques qu'on y aborde suscitent d'emblée l'intérêt d'un public de tout âge ${ }^{1}$. Que l'on songe à la trilogie romanesque de Dominique Demers, d'abord publiée au sein d'une collection pour la jeunesse au début des années $1990^{2}$; présenté par la suite dans une édition accessible au grand public, le roman Marie-Tempête, dont seul le paratexte $^{3}$ a été modifié, réunit en un volume les trois tomes de la série jeunesse. Reste qu'au sein de ces versions successives, l'hérö̈ne est une adolescente répondant au nom de Marie-Lune Dumoulin-Marchand, qui est âgée de quinze ans au moment où s'amorce le récit. Selon Jacques Allard, la trilogie de Dominique Demers constitue un roman de l'adolescence, "[de] ce type de l'âge difficile, si rare dans notre littérature, en fait souvent caché dans la case réservée des romans pour "jeunes" ${ }^{4}$. Suivant ce raisonne-

1. C'est pourquoi Danielle Thaler propose de penser les ouvres enfantines et juvéniles en termes de lecture. Sa rétlexion s'appuie sur le questionnement suivant: "Pourquoi [...] l'ecrivain qui songe à s'adresser aux enfants devrait-il bomer son horizon à un seul type de destinataire? . Voir Danielle Thaler, "Littérature de jeunesse: un concept problématique", Canadian Cbildren's Literature/Littérature canadienne pour la jeunesse (CCLLCD), Guelph (Ontario), n 83, 1996, p. 36.

2. Dominique Demers, In biver de tourmente, Montréal, La courte échelle, coll. "Roman + ", 1992 (puis Montréal, Québec/Amériquc, coll. "Tïan jeunesse", 1998); Les grands sapins ne meurent pas, Montréal, Québec/Amérique, coll. "Titan jeunesse ", 1993; Ils dansent dans la tempête, Montréal, Québec/Amérique, coll. "Titan jeunesse", 1994. On a décerné de nombreux prix à lauteure pour cette trilogie.

3. Il s'agit de tout ce qui figure en périphérie du texte à proprement parler: titre et couverture, dédicace, avertissement, préface, avant-propos, postface, etc. Voir Gérard Genette, Seuils, Paris, Seuil, coll. "Poétique ", 1987.

4. La préface de Jacques Allard montre bien la confusion qui prévaut lorsqu'il s'agit de distinguer le roman de l'adolescence du roman pour adolescents et adolescentes. Voir Dominique Demers, Marie-Tempête, préface de Jacques Allard, Montréal, Québec/Amérique, 1997, p. 9. 
ment, les romans qui s'adressent spécifiquement aux jeunes adultes et les romans thématisant l'adolescence sont comparables, voire identiques ${ }^{5}$. Il peut s'agir ainsi de romans d'apprentissage qui reproduisent à divers degrés l'évolution physiologique et psychologique de jeunes héros ou de jeunes héroünes. Comme le fait remarquer à juste titre Daniella Di Cecco, le roman de l'adolescence se distingue du Bildungsroman dans la mesure où "le protagoniste du roman de l'adolescence ne parvient pas nécessairement à s'intégrer socialement " $"$. À preuve, les romans de Colette, qui mettent en scène des jeunes filles vivant diverses expériences amoureuses, ne visent ni à glorifier ni à stigmatiser les conventions d'une société prescrivant à la femme les rôles traditionnels de mère et d'épouse. Un roman comme Le blé en berbe (1923) privilégie des figures d'adolescents dont le parcours ne se solde ni par une réussite ni par un échec sur le plan social. Âgés respectivement de 16 et de 15 ans, Vinca et Philippe, qui se connaissent depuis l'enfance, sont les acteurs principaux du récit. Au terme de vacances estivales qui se sont déroulées sur les plages paisibles de la Bretagne, les jeunes protagonistes font lapprentissage de l'amour et de la sexualité. À l'instar des jeunes gens de leur âge, les adolescents deviennent conscients des nouvelles pulsions qui les habitent. C'est pourquoi l'instance narrative insiste sur l'inexpérience d'un Philippe aux vues plutôt idéalistes. Tandis que le jeune homme appréhende les réactions de sa cadette devenue femme, il mythifie en quelque sorte la période précédant l'acte sexuel: "Il n'imaginait pas qu'un plaisir mal donné, mal reçu est une ceuvre perfectible. La noblesse du jeune âge l'entraînait seulement vers ce qu'il ne fallait pas laisser périr: quinze années de vie enchantée, de tendresse unique $[. . .]^{*}$." Par conséquent, l'été des jeunes adolescents illustre avec brio le

5. Entre 1900 et 1930 , faut-il le rappeler, la production des romans de ladolescence en France augmente considérablement et sollicite surtout un public adulte. Le Grand Meaulnes (1913) d'Alain-Fournier "n'a pas été destiné aux enfants, et pourtant, le public enfantin laa détourné à son usage ", en vertu de la présence de personnages adolescents, d'un espace propice aux aventures ainsi que de l'actualisation de thèmes propres aux contes de fée, tels le jeu et le mystère. Comme l'indique Élizabeth Ravoux-Rallo (Images de ladolescence dans quelques récits du Xx siècle, Paris, José Corti, 1989, p. 136-139), il serait intéressant de asavoir précisément pourquoi on assiste à la destruction systématique du roman pour la jeunesse à l'intérieur d'un roman pour aclulte".

6. Daniella Di Cecco, Entre femmes et jeunes filles. Le roman pour adolescentes en France et au Québec, Montréal, Éditions du remue-ménage, 2000, p. 27.

7. Colette, Le blé en berbe [1923], Paris, Éditions J'ai lu, 1974, p. 124. 
caractère inachevé du moi, la structure inchoative de l'identité, suivant en cela une perspective qui rappelle celle qu'adopte Henri Bergson relativement à la durée et à l'évolution créatrice. Il n'est guère besoin d'insister sur ce point : on connait l'influence déterminante qu'exerça Bergson sur les écrivains et les penseurs de l'époque à la suite, notamment, de l'accueil triomphal qui fut réservé à une conférence prononcée en $1911^{8}$.

Visiblement, la frontière existant entre, d'une part, les textes qui font de l'adolescence le coeur de leur propos et, d'autre part, ceux s'adressant aux adolescents et aux adolescentes pour en faire un destinataire de prédilection, demeure tellement floue au sein de la production contemporaine qu'il importe d'insister sur la pluralité des lectures qu'autorisent ces textes dominés par des figures de jeunes adultes. Dans le cadre de cette étude, il s'agit d'identifier quelques phénomènes textuels mis en ouvre par des romancières pour la jeunesse soucieuses d'élaborer des récits ne sollicitant pas nécessairement un seul type de destinataire. Des fictions romanesques dont les jeunes héroines sont engagées dans un processus d'écriture formeront l'essentiel du corpus étudié. En adoptant une telle posture énonciative, la jeune fille, on le verra, s'inscrit invariablement dans une dynamique favorisant la lecture et l'écriture, et qui est susceptible à ce titre de réviser et de corriger une épistémè patriarcale associant par définition le féminin au silence et à la passivité. Nombreuses, en effet, sont les ceuvres écrites pour la jeunesse qui procèdent d'un mouvement d'impureté romanesque dont le code est essentiellement dicté par des rapports intertextuels. Songeons à Marie-Lune, exaltée par le romantisme d'Alfred de Musset et la célèbre parabole du pélican dans la trilogie de Dominique Demers. Que dire également de Sara Lemieux, fidèle lectrice des Louisa M. Alcott, Anne Frank, Virginia Woolf et Marguerite Duras, et se retrouvant au cœur d'une odyssée qui la conduira jusqu'en Alaska'?

8. Il s'agit de la conférence intitulée "L'intuition philosophique ", présentée au Congrès de philosophie de Bologne. Le bergsonisme se répandit dès le début du $x^{t}$ siècle et forma toute une génération d'écrivains et de penseurs. Comme l'indique Ravoux-Rallo, "[le] triomphe dépassa vite les limites des cercles des spécialistes pour atteindre le grand public, et la jeunesse de l'époque, dans laquelle Bergson suscita de véritables vocations "; voir Images de l'adolescence dans quelques récits $d u \times x^{*}$ siècle, ouvr. cité. p. 31.

9. Anique Poitras, La lumière blanche (1993), La deuxième vie (1994), La chambre d'Éden I (1998) et La cbambre d'Ếden II (1998), Montréal, Québec/Amérique, coll. "Littérature jeunesse. ou "Titan jeunesse. (les deux noms d'une même collection). 
Pensons encore à la jeune Véronique parcourant à un rythme effréné des classiques américains tels Autant en emporte le vent (1938 en traduction française) de Margaret Mitchell et Sur la route (1960 en traduction française) de Jack Kerouac, alors qu'elle découvre l'amitié féminine ${ }^{10}$. À chaque fois, de pareilles figures de l'écrit rendent compte de "l'intertextualité [qui] compromet définitivement le caractère monolithique de la signification du texte littéraire: en introduisant un élément hétérogène, en faisant référence à un lieu de sens déjà constitué, elle brise toute univocité ${ }^{11}$ ".

Si le roman québécois pour la jeunesse depuis les années 1980 s'attarde au moi en devenir des adolescentes et au drame du corps sexué qui en résulte, à la manière des ouvrages de Colette, il fait appel également aux compétences encyclopédiques dont dispose le lectorat face à un texte se référant à d'autres textes: "La société arrive à enregistrer une information encyclopédique seulement parce que celle-ci a été fournie par des textes antérieurs ${ }^{12}$. C'est donc l'information insérée dans des œuvres pour la jeunesse constellées de signes de l'écrit qui fera l'objet de notre investigation. De fait, les romans destinés à un jeune public et parus depuis une vingtaine d'années au Québec illustrent de façon magistrale cette prolifération des savoirs questionnant la structure linéaire des textes. Aussi examinerons-nous des phénomènes discursifs qui, dans la mesure où ils établissent une filiation avec des textes antérieurs, requièrent, pour être correctement interprétés, "une compétence encyclopédique se [fondant] sur des données culturelles socialement acceptées en raison de leur "constance" statistique ${ }^{13}$ ". L'intertexte sert principalement, pensons-nous, à rendre compte du trajet identitaire de la jeune fille qui lit et qui écrit, en intégrant inéluctablement à son propos

10. Carole Fréchette, Do pour Dolorès, Montréal, La courte échelle, coll. "Roman ,+ 1999 .

11. Nathalie Piégay-Gros, Introduction à l'intertextualitê, Paris, Dunod, 1996, p. 74 .

12. Umberto Eco, Lector in fabula ou La cooperation interprétative dans les textes narratifis, traduit de l'italien, Paris, Grasset, 1985, p. 28. Dans Six promenades dans les bois du roman et d'ailleurs (traduit de l'anglais, Paris, Grasset, 1996), le même Eco invite un public non spécialiste à cerner l'importance du lecteur comme composante essentielle de la narration. De plus, l'ouvrage d'Eco montre l'intérêt des théories d'interprétation inspirées du parcours du lecteur de l'cuvre pour la jeunesse.

13. Umberto Eco, Lector in fabula, ouvr. cité, p. 19-20.

14. Umberto Eco, Lector in fabula, ouvr. cité, p. 21. 
des éléments de connaissance. Comme le soutient Eco, "il n'y a pas d'énoncé qui, pour être sémantiquement actualisé dans toutes ses possibilités de signification, ne requière un co-texte ${ }^{14}$. C'est manifestement ce co-texte (ou, si l'on préfère, cet intertexte) qu'il importe de décrire et d'analyser au sein du discours d'un je féminin scripteur, posé comme une composante essentielle du roman québécois pour l'adolescence.

Au Québec, le roman féminin de l'adolescence sort de son rigorisme moral lors de la parution des textes de Paule Daveluy à la fin des années 1950 et au début des années 1960. Nul doute que la production de l'écrivaine a contribué dans une large mesure à la transformation d'un discours romanesque hérité des modèles de la morale traditionnelle qui indiquait une voie à suivre aux adolescentes. Comme le précise Françoise Lepage, l'utilisation d'un je narrateur au sein de la série des "Rosanne", qui devait comporter trois volumes ${ }^{15}$, "témoigne de [l'avancée] de la parole adolescente ${ }^{16}$. L'été enchanté (1958) "ose pour la première fois peindre le monde tel que le voit une adolescente et permet une incursion dans la psychologie de son héroïne ${ }^{17}$ ". En rompant avec les thématiques passéistes et en adoptant le point de vue de la jeune fille moderne, les textes de Daveluy ouvrent la voie aux romans des années 1980 qui feront de l'adolescente un sujet désirant, doué de parole.

\section{L'avènement de la parole adolescente: Sylvette et la découverte de l'ailleurs}

Véritable pionnière du roman-miroir destiné à un public adolescent, Daveluy est également l'auteure de la série Sylvette qui comporte deux volumes: Sylvette et les adultes (1962) et Sylvette sous la tente bleue (1964) ${ }^{\text {lts. }}$. I.e texte qui relate les aventures de la

15. Trois romans: Lété enchanté (1958), Drôle d'attomne (1961) et Cet biver-là (1967).

16. Françoise lepage, "le concept d'adolescence: évolution et représentation dans la littérature québécoise pour la jeunesse. Voix et Images, Montréal, vol. $25, n^{\prime \prime} 2$, hiver 2000, p. $246-247$.

17. lrançoise lepage, "Le concept d'adolescence: évolution te représentation dans la littérature québécoise pour la jeunessen, art. cité.

18. Tous deux: Québec, Éclitions Jeunesse. Légèrement remaniés, ces deux ouvaages ont été rééclités: Montréal. Québec/Amérique, coll. "Littérature jeunesse", 1992 et 1993. Pour les fins de cette étude, nous aurons recours au 
jeune Sylvette Forest mérite une attention particulière, puisque le récit se présente entièrement sous la forme d'un journal intime, assignant d'emblée une fonction d'écriture à la narratrice. Les fréquents repères spatio-temporels servant à ramifier l'histoire évoquent la vie familiale et les expériences amoureuses d'une jeune fille issue d'une famille de trois enfants. Des segments narratifs réitérant le besoin de se dire scandent l'énonciation dun je féminin qui s'adonne à l'écriture. Ainsi, le récit de Sylvette et les adultes, débutant le 25 avril 1958 et se terminant le 7 juillet de la même année, est livré par une adolescente qui s'exprime à la première personne: "Parole de Sylvette! Cette fois, mon journal, je le tiendrai" (9). Il n'est guère étonnant que le je diariste, dès l'incipit, repousse toute comparaison avec les textes autobiographiques de Sigrid Undset et de Françoise Sagan ${ }^{19}$. Aux yeux de l'adolescente, l'acte de création se veut, bien entendu, original er unique. De pareilles allusions littéraires se greffent à des lexies porteuses de significations, dans la mesure où les énoncés se réfèrent d'emblée aux connaissances de l'héroïne, une collégienne âgée de 17 ans, vivant en banlieue de Montréal. D'ailleurs, la jeune fille avoue que le monde des humains l'inspire: "Comme les romanciers, je bâtis des histoires à partir des individuss" (28). Partant, des réflexions consacrées à l'univers compliqué des adultes nourrissent le discours de Sylvette qui établit un rapport de plus en plus concret avec le monde. A la suite des difficultés vécues par son père, employé dans un grand magasin où il doit sans cesse réclamer de l'argent aux clients endettés, la jeune fille se réfugie dans son univers où prédomine l'imagination: "La vie des adultes, quelle salade! Ce soir, je voudrais rentrer dans la clarté de ma petite enfance et n'en plus jamais sortir "(27), affirme Sylvette dans les carnets du 5 mai. La jeune fille demeure consciente du fait que les mots couchés sur le papier possèdent le pouvoir d'immortaliser les événements : "Je n'ai aucune prétention littéraire, puisque j'écris pour moi seule. Je défie le temps. Parfaitement. Je sais que ma mémoire est un sas. Rien ne s'y grave que l'immédiat ou l'extraordinaire. (33). Autrement dit, la

rexte original de Sylvette et les adultes, mais nous utiliserons aussi le texte publié chez Québec/Amérique. Les renvois aux textes seront indiqués par un chiffre entre parenthèses.

19. Dans la version remaniée, l'auteure a remplacé le nom de Sigrid Undset, écrivaine norvégienne qui s'est opposée farouchement à l'émancipation des femmes durant toute son existence, par celui de Marguerite Yourcenar. 
jeune fille a recours à l'écriture pour pallier les avatars de l'oubli. Sylvette se réfère de façon implicite à l'œuuve de Marcel Proust, célèbre pour avoir élaboré une philosophie du temps et de l'être: la protagoniste intègre de la sorte à son propos des énoncés qui ont trait à un passé qu'il importe de se remémorer. Alors qu'elle prépare ses examens de fin d'année, la jeune fille écrit, dans son journal en date du 16 juin: "J'use ma beauté en veilles épuisantes, pour récapituler, re-récapituler, à la recberche du temps perdu" $(115)^{20}$. Les savoirs littéraires, mis en discours par un je diariste sollicitant ses facultés mémorielles pour les fins d'un exercice scolaire, participent de l'autoreprésentation d'un personnage qui se perçoit tel un sujet pensant doué d'une conscience du temps. Rien d'étonnant alors si la narratrice rapporte les propos enflammés de son enseignante, qui se situent dans le prolongement d'un patriotisme ressortissant à un discours fondé sur "l'appel de la race». À l'occasion de la fête de Dollard des Ormeaux, sceur Sainte-Gertrude cite l'historien Arnold J. Toynbee qui a vu en "la terre d'espérance, de jeunesse et de liberté" qu'incarne l'Allemagne "the coming people of the century" (70) ${ }^{21}$.

Bien que l'école et les problèmes familiaux lui réclament beaucoup de son temps, Sylvette songe souvent aux garçons. Au désespoir de sa mère, elle se met à fréquenter un jeune homme qui habite à proximité de chez ses parents. Il s'agit de Jean-Pierre, "aux yeux d'ombre et aux cheveux de nuit" (81). Reproduisant les stéréotypes et les idées reçues de la société où elle est confinée, la jeune fille a tôt fait de se comporter comme les hérö̈nes du grand écran, aux allures de princesse: "passant devant chez lui, tout à l'heure, je laissai choir mes livres de bibliothèque" (65). Cependant, les relations amoureuses n'ont rien à voir avec les productions hollywoodiennes, comme le confie une Sylvette penaude à son journal: "Mon premier baiser, c'est raté! Moi qui brossais un décor de rêve, comme dans les films en technicolor: un visage

20. C'est nous qui soulignons.

21. Toynbee (A Study of History, nouvelle édition, Londres, Oxford University Press, 1972) a utilisé le terme postmodernisme en 1947 pour désigner le nouveau cycle historique dans lequel s'engageait la société occidentale. Ce cycle aurait commencé en 1875 , au moment où triomphe la puissance impérialiste de l'Angleterre, et aurait atteint son apogée en 1902 à la suite de la victoire lors de la guerre des Boers. Le danger de l'impérialisme, selon Toynbee, réside dans la destruction de la créativité souvent générée par les jeunes générations. 
mâle tremblant d'êmoi, des lèvres posées sur les miennes avec une tendre gravité" (90-91). Au fil du temps, l'adolescente finit par comprendre que les amants peuvent commettre des maladresses puisqu'ils vivent dans un univers perfectible. D'ailleurs, l'héroïne apprend à mieux connaître sa mère qui devient progressivement une amie et une confidente: "nous causions, maman et moi, dans le calme de la nuit naissante [...] et je posai des questions d'ordre intime" (131). Dans un contexte propice au dialogue survient pourtant un événement tragique : la mère de Sylvette meurt noyée dans un lac. L'épilogue, qui s'attarde au deuil que vivent pendant plusieurs mois le mari et ses enfants, laisse toutefois présager un dénouement heureux pour la famille Forest qui accueillera en son giron un nouveau membre: Geneviève Brabant, la future fiancée du père de Sylvette. De fait, la jeune femme accompagnera la famille lors de son excursion en Gaspésie.

Le voyage campe l'action de Sylvette sous la tente bleue, qui est la suite chronologique du premier roman et qui s'échelonne du 13 juillet au 7 août 1959. Captivée par la présence de l'autre en elle, Sylvette, maintenant âgée de 19 ans, décide de s'improviser comédienne. Tout au long du voyage où elle se rend en Gaspésie avec sa famille, la jeune fille s'évertue à répéter le rôle du personnage d'Hélène figurant dans Le songe d'une nuit d'été de William Shakespeare: "Et vivent ces lignes à apprendre, rythmées, difficiles, d'où jaillira une autre Sylvette" (25). L'adolescente aspire résolument au changement dans la mesure où elle se perçoit comme dépendante de l'opinion publique et plutôt conformiste : La mer m'attire. Je sais qu'après l'avoir connue, je ne serai plus jamais, ensuite, tout à fait la même" (16). Si la protagoniste néglige quelque peu la rédaction de son journal intime, c'est pour mieux apprécier le paysage pittoresque qui défile sous ses yeux: "Je suis en retard dans mon journal; à peine si j'ai le temps d'écrire tellement il y a de beautés à admirer" (127). Tout se passe comme si le texte de Shakespeare, que la jeune fille traîne partout avec elle, devenait le co-texte à partir duquel émanent les significations d'un récit où vont s'entrecroiser les intrigues amoureuses. Précisons que l'intérêt de la jeune fille pour l'art dramatique va l'amener à rencontrer des artistes et des comédiens, puis à se produire sur la scène du Centre d'art de Percé. Un homme qu'elle a remarqué au cours du trajet lui demande de remplacer au pied levé la comédienne qui devait justement interpréter le rôle d'Hélène dans Le songe d'une nuit d'été. Éprise momentané- 
ment d'Éric Landreville surnommé le "Viking blond" (150), qui joue le rôle de Démétrius au théâtre d'été de Percé, Sylvette fait siennes les paroles de l'Athénienne: "Je suis à vous, Démétrius/Comme un épagneul à son maitre/Négligez-moi, repoussez$\mathrm{moi} /$ Et s'il faut que vous me frappiez/Frappez-moi. Je rampe à vos pieds" (93). Au sein des carnets, la fascination qu'exerce Éric sur la jeune fille est associée à un philtre magique, comme on en retrouve à souhait dans l'histoire du Songe. À l'exemple d'Hélène, Sylvette se complaît à jouer les héroïnes blessées et se confond avec son personnage, puisqu'elle est amoureuse de Démétrius/Éric: "Soleil, éclairez-moi/ces routes incertaines/Afin que je retrouve Athènes/Loin de ce coeur cruel qui me raille ou m'évite/Ah dormir!...Viens sommeil. Endors ma peine extrême/Et fais-moi m'oublier moi-mêmen. Sans contredit, le thêâtre permet à Sylvette de rompre avec la figure conventionnelle de la jeune banlieusarde soumise, timorée, cantonnée dans la routine. Bref, une image de la femme que projette, à certains égards, le premier tome de la série. Plus encore, le discours citationnel qui reproduit des fragments du Songe d'une nuit d'été se conjugue au parcours spatial de l'héroïne appelée à effectuer une traversée inattendue du domaine d'Éros et devient ainsi la métaphore du temps et du mouvement. Suite au périple en Gaspésie, d'autres illusions se sont envolées puisque Sylvette prend conscience que les princes charmants sont des êtres évanescents, comme en témoigne l'attitude d'Éric pressé d'aller rejoindre son épouse fortunée. Bien que la loi du père se fasse difficilement oublier dans le Québec des années 1950, il n'en demeure pas moins que la jeune narratrice parvient à s'exprimer comme un sujet amoureux au fil de son journal et au théâtre.

\section{Une parole adolescente autonome : Cassiopée et les choix de l'amour}

Force est de constater que les récits pris en charge par des adolescentes dans un contexte d'énonciation où le je féminin entreprend de rédiger un journal intime donnent lieu à des figures de l'écrit modélisant le devenir des héroïnes. Souvent comparés par la critique aux romans de Paule Daveluy, les textes de Michelle Marineau, dont la jeune Cassiopée Bérubé-Allard est le personnage principal, en constituent la preuve éloquente. Publiée à la fin des années 1980, la série Cassiopée est composée de deux 
romans: Cassiopée ou L'été polonais et L'été des baleines ${ }^{22}$. Âgée de 15 ans, Cassiopée devient amoureuse d'un jeune Polonais qu'elle a rencontré lors d'une fugue à New York, au début des vacances scolaires. Relaté à la première personne, le texte emprunte la forme du journal intime. Dès les premières lignes, la narratrice informe le lecteur de son entreprise: "J'ai pris mon beau cahier bleu à petits carreaux et je me suis mise à écrire tout ça" (26). Afin d'identifier les repères encyclopédiques qui soustendent le texte de la diariste, il importe de rappeler les paroles de la chanson Je voudrais voir la mer (1986), dont un extrait est mis en exergue. Ces paroles, écrites par Michel Rivard, disent le désir d'un être qui, vivant en ville, aimerait se confondre avec les paysages marins. Il y est question notamment des "plages d'argent ", des "oiseaux de lune", des "nuages de laine", du "mouvant de la vague". On sait que l'action principale du récit des aventures de l'adolescente se déroule au chalet de la famille de Marek, situé en bordure de l'océan, sur une île. C'est en effet sur les plages du Rhode Island que Cassiopée vit ses premières expériences amoureuses. L'imaginaire marin évoqué dans la chanson représente un univers de prédilection pour les jeunes tourtereaux, puisque Marek et sa douce entreprendront l'été suivant un voyage au pays des baleines, dans le Nord du Québec. Des renvois à la poésie de Jacques Prévert ponctuent également le discours de la jeune fille qui s'adonne à la lecture lors de ses vacances à la mer: "Je découvre, émerveillée, des mondes dont je ne soupçonnais pas l'existence [...] Démons et merveilles... Je ferme les yeux et je me répète ces lignes, encore et encore, comme une incantation" (144-145). Les vers cités par l'adolescente, évoquant derechef les mondes possibles de la mer, proviennent du poème "Sables mouvants" qui fait partie du recueil Paroles (1945). Il s'agit d'un poème où le sujet de l'énonciation s'adresse vraisemblablement à un $t u$ abandonné au sommeil. Le réseau métaphorique institué traduit le sentiment amoureux du je à l'endroit d'un être qui "[...] remue en rêvant/Démons et merveilles/Vents et marées". Le séjour de Cassiopée aux États-Unis lui fait découvrir de nouveaux univers poétiques, alors qu'elle prend conscience du pouvoir des mots et de l'imagination. Si l'adolescente repro-

22. Tous deux: Montréal, Québec/Amérique Jeunesse, 1988 et 1989 . Les renvois à cette édition seront indiqués par un chiffre entre parenthèses. On a décerné quelques prix à l'auteure pour cette série, dont le prix du Gouverneur général. 
duit au sein de son journal des extraits de poésie et de chanson, elle est également amenée à interpréter un dessin que lui a fait parvenir son oncle Jean-Claude complétant des études cinématographiques à New York. Ce dessin est de Marcel Duchamp et s'intitule Avoir l'apprenti dans le soleil (1914). En s'inscrivant dans le cycle de la rotation et du mouvement caractéristique de la Roue de bicyclette (1913), c'est-à-dire de l'un des readymade les plus célèbres de l'artiste, il pourrait correspondre à un bref poème dont la beauté se déploie à travers le syntagme "Icare et ses clisciples" apparaissant en retrait sur la missive expédiée à Cassiopée. Quoique ladolescente ne soit pas en mesure de cerner toute la portée du dessin et du texte qui l'accompagne, elle affirme que l'illustration de la bicyclette renvoyant à des signifiés de lumière et de mouvement "[lui] a fait faire des tas de pirouettes dans [sa] tête" (41). Nul doute que l'activité d'écriture de Cassiopée prend appui sur un régime d'intertextualité favorisant l'inscription du sujet féminin dans les sphères du savoir.

Le récit de L'été des baleines s'amorce au moment où la jeune fille renoue avec l'écriture. De retour à Montréal, l'adolescente poursuit la rédaction de son journal entrepris l'été précédent: "Alors je reprends mon journal, auquel je n'ai pas touché depuis cinq mois. Depuis mon séjour au bord de la mer. Depuis ce que j'ai appelé mon été polonais" (15-16). Au fil de ses échanges avec Marek vivant toujours à New York, le je diariste intègre à son récit une dimension poétique puisée à même les textes de Paul Eluard dont la tendresse pour les êtres et les choses semble infinie: "Le front aux vitres comme font les veilleurs de chagrin/Je te cherche pardelà l'attente/Par delà moi-même/Et je ne sais plus tant je t'aime/Lequel de nous deux est absent" (30-31). Les vers reproduits par l'adolescente sont tirés de Liamour la poésie (1929). Le texte qui s'articule autour du thème de l'amour et du vide généré par l'ahsence de l'être aimé témoigne explicitement de la situation de Cassiopée, séparée pour la longue période hivernale de son amoureux par des milliers de kilomètres. Il importe de souligner que l'adolescente a découvert la poésie d'Éluard par l'entremise de sa mère, laquelle lui a fait présent d'un recueil à l'occasion de Noël: "Les poèmes d'Eluard sont beaux partout et en tout temps, mais peutêtre encore plus quand on a quinze ans et qu'on est amoureuse" (29). Pareille modélisation narrative de figures de l'écrit procède d'une généalogie féminine émanant de la transmission d'un savoir. Émues toutes deux par une poésie aux accents lyriques, la mère et 
la fille n'en sont que plus liées. Or, ce savoir littéraire n'est point utilisé à des fins moralisatrices par l'aînée qui, à aucun moment, n'adopte l'attitude du parent voulant contrôler et dominer sa progéniture. Au contraire, la mère encourage sa fille à être autonome et à exercer sa liberté d'action lors de ses retrouvailles avec Marek qui se propose de passer l'été auprès d'elle. Des poèmes de Gaston Miron surgissent du récit du personnage féminin qui, sur la route la conduisant à l'aéroport, se remémore des extraits de "La marche à l'amour ${ }^{23}$ ": "tu es mon amour/ma clameur mon bramement/je marche à toi, je titube à toi/je meurs de toi "(113-114). Soulignons que le compagnon de Cassiopée accuse les traits du jeune homme romantique, se disant poète et désirant connaitre la poésie issue du pays de sa douce. Il n'est point étonnant non plus que la narratrice étaye son journal de bord de fragments de la poésie de Gatien Lapointe, alors qu'elle entreprend une longue excursion vers le nord: "J'ai toute la confusion d'un fleuve qui s'éveille" (123) ${ }^{24}$.

Or, le journal de la jeune fille ne cite pas uniquement des textes d'auteurs masculins. Il inclut des références à l'ouvre de Marie Uguay et Marie Savard, deux poètes québécoises, amorçant en effet un dialogue avec leurs textes. Extraits de Signe et rumeur (1976) ${ }^{25}$, les vers de Marie Uguay inaugurent le journal de l'adolescente: "L'hiver nous retire vers la mémoire. (15). Le propos intimiste est pris en charge par un je d'énonciation féminin sollicitant un destinataire. En voici un exemple: "Hors de toi le temps est une prolongation difficile ${ }^{26}$." Si l'hypotexte mis en exergue circonscrit le cadre spatio-temporel du récit, il préfigure également un discours axé sur la mémoire et l'identité. Tout se passe comme si la jeune Cassiopée devait préserver le souvenir de Marek au fil de l'hiver passê loin de lui et ce, au moyen de l'écriture. Tandis que s'achève le récit du voyage des tourtereaux à Longue-Pointe, où l'on peut admirer les baleines, surviennent les vers de Marie Savard, extraits de Sur l'air d'Iphigénie (1984) ${ }^{27}$ : "Je

23. Gaston Miron, L'bomme rapaillé, Montréal, Presses de l'Université de Montréal, 1970.

24. Gatien Lapointe, Ode au Saint-Laurent précédée de J'appartiens à la terre, Montréal, Éditions du Jour, coll. "Les poètes du jour", 1963.

25. Marie Uguay, Signe et rumeur, Saint-Lambert, Éditions du Norôt, 1976.

26. Marie Uguay, ouvr. cité, s.p.

27. Marie Savard, Sur l'air d'lpbigénie, théâtre, Montréal, Éditions de la Pleine lune, 1984. Rappelons que Marie Savard a fondé en 1974 les Éditions de la Pleine lune, la première maison d'édition de femmes au Québec. 
ne sais plus très bien ce que j'attends/de moi/ou qui j'attends/mais je sais que la terre s'égare sous mes pas" (189). Luimême composé de renvois à des textes féministes ${ }^{28}$, le drame poétique dont Savard est l'auteure est constitué d'un dialogue entre une mère et sa fille, tournant en dérision l'imaginaire patriarcal. L'hypotexte, dont les signifiés renvoient au doute et à l'incertitude, rend compte des sentiments ambivalents de la jeune fille, amoureuse de deux garçons. On comprend que le "second été polonais" vécu par l'héroïne dans le Nord québécois donne lieu à une quête réalisée suivant les principes de l' agentivité ${ }^{24}$ ": l'adolescente doit procéder à des choix existentiels et, bien entendu, les assumer, quelles qu'en soient les conséquences. En pareil cas, l'agentivité consiste précisément à agir autrement qu'en conformité avec les préceptes prescrits par le statu quo, afin de se construire une identité cohérente et d'agir en accord avec ses valeurs et ses désirs. L'excursion à Longue-Pointe aura certes permis à Cassiopée d'exprimer un discours amoureux au moyen de son propre langage. Toutefois, on remarquera que c'est d'abord à travers les figures de l'écrit qui jalonnent son récit que le je féminin s'actualise comme être autonome.

\section{Une parole adolescente hybride: Sara et la généalogie féminine}

À l'instar de Sylvette Forest qui s'apprête à jouer dans une pièce de Shakespeare, Sara Lemieux, l'héroïne de la tétralogie d'Anique Poitras, s'approprie le rôle de Juliette alors qu'elle est invitêe à se joindre à une troupe de théâtre amateur. Au sein des deux premiers tomes intitulés respectivement La lumière blanche et La deuxième vie ${ }^{30}$, l'hypotexte Roméo et Juliette de William Shakespeare devient un lieu discursif où la fiction et la réalité se confondent dans la pensée de celle qui a vu mourir sous ses yeux son

28. Il s'agit notamment de l'ouvrage de Luce Irigaray, Le conps-à corps avec la mère, Montréal, Éditions de là Pleine June, 1981.

29. Judith Butler a développé le concept d'agentivité * qui renvoie aux actions des femmes ne souscrivant pas aux systèmes de références d'une pensée patriarcale. Voir Judith Butler, Gender Trouble. Feminism and the Subtersion of ldentity, New York et Londres, Routledge, 1990.

30. Anique Poitras, ouvr. cité. Dorénavant, les renvois aux textes seront indiqués par le numéro de page entre parenthèses, précédé des abréviations $L B$ pour $L a$ lumière blanche, DV pour La deuxième vie et CE pour La chambre d'Éden $I$. 
premier amoureux: "J'ai douze ans et mon amour est mort" ( $L B$, 141). C'est à Juliette Capulet que s'identifie Sara Lemieux après avoir été séparée de Serge Viens, son Roméo: "je lis et je relis Roméo et Juliette de William Shakespeare. Cela m'apaise" $(D V, 18)$. Bien que la tragédie date du $x v v^{e}$ siècle, elle relate des faits qui n'ont rien d'obsolète aux yeux de l'adolescente. Suivant la perspective de la narratrice, la fiction où elle s'est introduite contribue à prolonger la réalité de l'amour incarné par un être disparu prématurément. Par le biais du travail de la citation, l'œuvre théâtrale conçue dans l'Angleterre du Xvi ${ }^{e}$ siècle, puis jouée quatre siècles plus tard à Montréal, a pour fonction de traduire l'expérience tragique d'une jeune fille vivant dans le Québec contemporain. Thématisant la passion de deux adolescents, la pièce comporte une dimension symbolique pour l'héroïne. Le je féminin devient pour ainsi dire Juliette Capulet, à l'exemple de Sylvette métamorphosêe en un personnage du Songe d'une nuit d'été afin de donner la réplique à un indéfectible séducteur sur les plages de Percé. Tout se passe comme si les textes de Daveluy avaient ouvert la voie aux romans hybrides de la postmodernité et, du même élan, constitué une filiation à partir de laquelle va s'inventer l'écriture au féminin. À l'image de Sylvette s'inscrivant dans un processus de lecture et d'écriture, Sara Lemieux est associée à un espace dominé par les arts et la culture. Si l'héroïne de Daveluy s'adonne au théâtre amateur et se raconte à travers un journal intime, Sara Lemieux a également l'occasion de promouvoir ses talents de comédienne et d'écrivaine. À la veille de ses quinze ans, l'adolescente se consacre de façon assidue à l'écriture en tenant un journal, à la suite du message que lui a livré sa mère agonisante sur une bande sonore. La défunte a légué à sa fille un livre "[qu'elle a] toujours gardé aussi précieusement que la prunelle de [ses] yeux, puisqu'il avait appartenu à [sa] mère" $(C E, 22)$. Il s'agit du Joumal (1950 en traduction française) d'Anne Frank. Si le texte évoque à nouveau la figure d'une adolescente, il constitue aussi une marque de la filiation à laquelle s'identifie Sara. À la manière d'Anne Frank, Sara Lemieux devient un je diariste: "J'ai lu le Journal d'Anne Frank. Cette fille de mon âge est morte à quinze ans dans un camp de concentration pendant la Deuxième Guerre mondiale. C'est à cause d'elle que j'ai décidé de tenir mon journal moi aussi", écrit Sara le 2 juillet depuis Toronto, où elle vit avec son père $(C E, 23)$. Fondée sur l'intertextualité, une telle pratique d'écriture fait intervenir, d'une part, plusieurs codes qui font éclater les cadres conventionnels du genre romanesque et, d'autre part, met 
en scène un je féminin déterminé à laisser libre cours à ses désirs et à ses rêves. Comme le précise Valérie Raoul, les effets de miroir caractérisent la forme du journal:

le diariste se contemple, et pas toujours favorablement. Il projette une image de lui-même en mots, mais là ne s'arrête pas son rôle de projection et de réflexion. Il ne se voit pas seulement, il est également conscient que les autres le regardent, qu'il regarde les autres, et enfin qu'il se regarde lui-même ${ }^{31}$.

L'utilisation que font les écrivaines du journal fictif comme procédé de représentation des protagonistes féminines demeure, en somme, révélatrice d'un processus d'individuation généré par les figures de l'écrit, elles-mêmes associées à des lieux du savoir.

À l'intérieur des récits faisant l'objet de nos investigations, l'intertextualité sert de catalyseur aux narratrices désirant créer un espace dans lequel il leur sera possible de "s'affranchir des liens qui immobilisent leur pensée, leur corps, leur imagination ${ }^{32}{ }^{\text {* }}$ Les je diaristes animés d'un désir de connaissance cherchent à s'affranchir d'une pensée logocentrique édifiée sur la domination et l'autorité. Les romans destinés à un public adolescent qui ont retenu notre attention préfigurent et actualisent tout à la fois les croisements littéraires du postmodernisme et du féminisme à travers les formes discursives de l'intertextualité, elle-même instigatrice de la pratique autoreprésentative d'un sujet féminin qui fait advenir un dialogue fécond entre des textes de provenance générique diverse et qui développe ses propres systèmes de références. Certes, l'incidence d'une critique postmoderne, renonçant à une vision totalisante du savoir et à une pratique du discours unifié, sur une pensée féministe cherchant à "transgresser la forme d'écriture sociale imposée a priori ${ }^{33}$ " est manifeste: "Fécond, inventif, varié, le discours féministe québécois [a enrichi] la fac-

31. Valéric Raoul, Le joumal fictif dans le roman francais, Paris, Presses universitaires de France, 1999, p. 82.

32. Marisa Zavalloni, "Introduction", clans Marisa Zavalloni (sous la dir. de), L'émergence d'une culture au féminin, Montréal, Les Éditions Saint-Martin, 1987, p. 7.

33. Evelyn Voldeng, "Lintertextualité dans les êcrits féminins d'inspiration féministe", Voix et lmages, Montréal, vol, 7, n' 3, 1982, p. 528. 
Lucie Guillemette

ture du postmodernisme d'une forte dimension éthique ${ }^{34}$ ", prétend Janet Paterson. À la lumière de notre analyse s'élabore un paradigme de l'écriture féminine pour la jeunesse marquée de l'hybridité postmoderne et de l'agentivité féministe. À titre de narratrice à la première personne, la jeune fille qui lit et qui écrit constitue une composante essentielle du roman québécois de l'adolescence, lequel sollicite un lectorat dont les compétences encyclopédiques lui permettent d'interpréter les textes au fur et à mesure qu'ils passent de la fonction didactique à la fonction esthétique.

34. Janet M. Paterson, "Le postmodernisme québécois: tendances actuelles", Etudes littéraires, Québec, vol. 27, n 1, êté 1994, p. 77. 